\title{
Seminal Vesicle Biopsy
}

National Cancer Institute

\section{Source}

National Cancer Institute. Seminal Vesicle Biopsy. NCI Thesaurus. Code C94512.

The removal of tissue from the seminal vesicles for the purpose of microscopic evaluation. 